\title{
Árvores de decisão baseadas em entalpia para avaliação de conforto para poedeiras
}

\section{leves em clima tropical}

\author{
Enthalpy-based decision trees for comfort assessment of light layers in a tropical environment \\ Árboles de decisión basados en la entalpía para la evaluación del confort de las ponedoras en clima \\ tropical
}

Recebido: 26/02/2021 | Revisado: 06/03/2021 | Aceito: 16/03/2021 | Publicado: 20/03/2021

\author{
Érik dos Santos Harada \\ ORCID: https://orcid.org/0000-0001-9851-2722 \\ Universidade Estadual Paulista, Brasil \\ E-mail: erik.harada8@gmail.com \\ Maria Elena Silva Montanhani \\ ORCID: https://orcid.org/0000-0003-1987-7366 \\ Universidade Estadual Paulista, Brasil \\ E-mail: maria.montanhani@gmail.com \\ Leda Gobbo de Freitas Bueno \\ ORCID: https://orcid.org/0000-0001-8188-0000 \\ Universidade Estadual Paulista, Brasil \\ E-mail: leda.bueno@unesp.br \\ Mario Mollo Neto \\ ORCID: https://orcid.org/0000-0002-8341-4190 \\ Universidade Estadual Paulista, Brasil \\ E-mail: mario.mollo@unesp.br \\ Silvia Regina Lucas de Souza \\ ORCID: https://orcid.org/0000-0001-9575-4355 \\ Universidade Estadual Paulista, Brasil \\ E-mail: silvia.souza@unesp.br \\ Ricardo da Fonseca \\ ORCID: https://orcid.org/0000-0002-1163-6296 \\ Universidade Estadual Paulista, Brasil \\ E-mail: ricardo.fonseca@unesp.br
}

\begin{abstract}
Resumo
Com o avanço da tecnologia foi possível armazenar uma grande quantidade de dados com um menor preço, sendo possível investigar os fatores climáticos que afetam a produção animal. A região Oeste do Estado de São Paulo é uma região propensa à ocorrência de extremos de temperatura, sendo um fator preocupante para os produtores de ovos. Neste estudo objetivou-se saber se é possível gerar árvores de decisão a partir de dados de entalpia utilizando a Mineração de Dados, e se estas árvores estarão aptas a serem inseridas em um sistema de alerta para intempéries climáticas. Para este estudo foi utilizado um banco de dados de variáveis bioclimáticas provenientes de três aviários de poedeiras comerciais localizados na cidade de Bastos-SP, Brasil, no ano de 2013. Após a organização e classificação dos dados em planilha eletrônica, e processados pelo software Weka $\square$ com o algoritmo J48 (C4.5) para mineração de dados, a técnica aplicada a este banco de dados possibilitou a geração de árvores de decisão com aproximadamente $98 \%$ de acertos e índice Kappa de 0,96 respectivamente. Sendo assim, as árvores de decisão geradas neste estudo são precisas o suficiente, para que sejam utilizadas futuramente em um sistema de alerta contra adversidades climáticas para poedeiras comerciais em clima tropical.
\end{abstract}

Palavras-chave: Extremos climáticos; Mineração de dados; Avicultura de postura.

\begin{abstract}
With the advancement of technology, it was possible to store a large amount of data at a lower price, making it possible to investigate the climatic factors that affect animal production. The western region of the state of São Paulo is a region prone to extremes of temperature, which is a worrying factor for egg producers. This study aimed to find out if it is possible to generate decision trees from enthalpy data using Data Mining, and if these trees are suitable to be inserted in a weather forecast system. For this study, a database of bioclimatic variables from three commercial layer aviaries located in the city of Bastos-SP, Brazil, in the year 2013 was used. After organizing and classifying the data in a spreadsheet, and processed by Weka $\square$ software with the J48 algorithm (C4.5) for data mining, this technique applied to this database allowed the generation of decision trees with approximately $98 \%$ and 0.96 for Kappa index, respectively. Thus, the decision trees generated in this study are accurate enough to be used in a future warning system against climatic adversities for commercial layers in tropical climates.
\end{abstract}


Keywords: Climate extremes; Data mining; Layer poultry.

\section{Resumen}

Con el avance de la tecnología fue posible almacenar una gran cantidad de datos con un precio menor, siendo posible investigar los factores climáticos que afectan a la producción animal. La región occidental del estado de São Paulo es una región propensa a la ocurrencia de temperaturas extremas, lo que constituye un factor preocupante para los productores de huevos. En este estudio, el objetivo era saber si es posible generar árboles de decisión a partir de datos de entalpía utilizando la Minería de Datos, y si estos árboles serán adecuados para ser insertados en un sistema de alerta meteorológica. Para este estudio, se utilizó una base de datos de variables bioclimáticas de tres granjas comerciales de ponedoras ubicadas en la ciudad de Bastos-SP, Brasil, en 2013. Después de organizar y clasificar los datos en una hoja de cálculo electrónica, y procesados por el software Weka $\square$ con el algoritmo J48 (C4.5) para la minería de datos. Esta técnica aplicada a esta base de datos permitió generar árboles de decisión con aproximadamente un 98\% de respuestas correctas y un índice Kappa de 0,96 respectivamente. Por lo tanto, los árboles de decisión generados en este estudio son lo suficientemente precisos como para ser utilizados en el futuro en un sistema de alerta contra adversidades climáticas para capas comerciales en climas tropicales.

Palabras clave: Extremos Climáticos; Minería de datos; Aves de Corral ponedoras.

\section{Introdução}

A ocorrência de ondas de calor tem se tornado frequente, e as mesmas têm causado efeitos negativos a produção animal. No ano de 2020, o Instituto Nacional de Meteorologia- INMET, emitiu alertas sobre a severidade de uma onda de calor com $5^{\circ} \mathrm{C}$ acima da média por um período de entre 3 e 5 dias, alerta que incluía uma importante região produtora de ovos localizada no Oeste do Estado de São Paulo (INMET, 2020). Existe uma preocupação mundial em relação à produção de alimentos. Estimase, que em 2050 haja uma redução de $38 \%$ a $57 \%$ na produção de milho em decorrência de ondas de calor e longos períodos de estiagem (Chung et al., 2014). Segundo Salgado \& Nääs (2010), a região de Bastos-SP é um local propenso à ocorrência de temperaturas extremas diárias durante o ano, possuindo aproximadamente $29 \%$ de risco de ocorrência de temperaturas extremas durante o ano, fato preocupante, pois o município tem como principal atividade econômica a avicultura de postura.

A produtividade máxima das galinhas poedeiras é atingida quando as mesmas são inseridas em um local que conceda as exigências de bem-estar, pois este estado reflete na saúde física e mental, e conforto térmico para as aves (Andrade et al., 2018). Para que seja possível a realização de tal atividade, averíguam-se constantes investimentos em melhorias na produtividade. A mesma se encontra associada a alguns fatores importantes: melhoramento genético, investimento nas instalações, treinamento dos funcionários em relação ao manejo, melhoria nas condições sanitárias entre outros. As variáveis ambientais externas causam interposição nas condições internas da instalação, podendo causar infortúnios a produção, acarretando dispêndios econômicos à atividade (Oliveira \& Nääs, 2012).

O estresse térmico por calor ocasiona sérios problemas fisiológicos, afetando o sistema endócrino, respiratório e o balanço eletrolítico. Todos esses distúrbios afetam negativamente o desempenho destes animais (Teeter et al., 1985; Sohail et al., 2010; Lara \& Rostagno, 2013). As causas do estresse térmico por calor são provocadas pela associação de altas temperaturas, altas taxas de umidade relativa do ar e baixa velocidade do vento (Yahav et al., 2004; Balnave \& Brake, 2005). Para que os efeitos do calor sejam minimizados, o sistema de termorregulação das aves é acionado quando a temperatura ambiente está acima de $24^{\circ} \mathrm{C}$, temperatura esta, acima da zona de termoneutralidade (Celik et al., 2004; Etches et al., 2008). O estresse por calor se torna visível, quando a temperatura passa de $30^{\circ} \mathrm{C}$, pois, devido à dificuldade em dissipar calor pela ausência de glândulas sudoríparas e à presença de penas, este torna-se mais evidente (Seven, 2008). Sabe-se também que o estresse por calor favorece a diminuição do peso corporal e negativamente a produção e qualidade dos ovos, este pode também ocasionar elevação da taxa de mortalidade (Pereira et al., 2010).

Perante à necessidade de monitorar o ambiente, foram correlacionados dados ambientais como: temperatura, umidade relativa, velocidade do vento entre outros, podendo contribuir para a elaboração de Índice de Conforto Térmico. As variáveis de temperatura do ar, umidade relativa, velocidade do vento e radiação solar afetam o desempenho das aves (Medeiros et al., 2005; 
Damasceno et al., 2010). Utilizando apenas temperatura e umidade relativa, o índice de Entalpia é bastante utilizado para classificar o ambiente e o desconforto animal, pois são variáveis ambientais disponíveis facilmente em estações meteorológicas (Guimarães et al., 2014).

Junto com a evolução da cadeia produtiva, o grande volume de dados armazenados que são coletados dos processos produtivos, também aumenta a cada ano. Em decorrência da evolução tecnológica, o armazenamento destes dados em bancos de dados digitais teve uma redução nos preços, tornando-o acessível. Para que seja possível interpretá-los com melhor aproveitamento, são necessárias técnicas e recursos computacionais (ANID, 2015). Entre os recursos disponíveis, a Mineração de Dados é uma ferramenta que associa inteligência artificial e ciência computacional, gerenciando os dados, algoritmos matemáticos e a estatística, possibilitando analisar um grande volume de dados (Liao, 2003; Han \& Kamber, 2006). Desta forma, para melhor prever as adversidades climáticas, a ferramenta de mineração de dados com a geração de árvores de decisão é uma alternativa que auxilia no entendimento da ação do clima em relação às instalações para animais.

Com o presente cenário, o objetivo deste estudo é desenvolver árvores de decisão com alta precisão a partir de dados de entalpia, servindo de base para um sistema de alerta contra adversidades climáticas para poedeiras leves em clima tropical.

\section{Materiais e Métodos}

Este estudo foi aprovado pela Comissão de Ética em Uso de Animais (CEUA) da FCAT/Unesp sob o número de protocolo 11/2012.

Para este estudo, foram utilizados três aviários comerciais, que se diferenciavam tipologia de construção: sistemas de climatização (piramidal sem climatização, vertical com pressão negativa e vertical com aspersão no telhado), denominados respectivamente A1, A2 e A3. Os aviários eram localizados no município de Bastos-SP (latitude 21 ${ }^{\circ} 55^{\prime} 19^{\prime \prime}$ sul e longitude $50^{\circ} 44^{\prime} 02^{\prime \prime}$ oeste, altitude de 445 metros). O clima da região é subtropical Aw (tropical chuvoso com inverno seco e mês mais frio com temperatura média superior a $18^{\circ} \mathrm{C}$ (CEPAGRI, 2017). O mês mais seco tem precipitação inferior a $60 \mathrm{~mm}$ e com período chuvoso que se atrasa para o outono.

O estudo foi dividido em duas partes e, na primeira, houve as coletas das variáveis bioclimáticas (Temperatura de Bulbo Seco $\left({ }^{\circ} \mathrm{C}\right)$ e Umidade Relativa do $\operatorname{Ar}(\%)$ ), esta etapa ocorreu no ano de 2013 para os três tipos de aviário, a segunda parte foi a realização da Mineração de Dados. Utilizaram-se poedeiras da linhagem Dekalb White ${ }^{\circledR}$, com 17 semanas de vida, todas recebendo alimentação equivalente, o período se iniciou em março de 2013.

\subsection{Monitoramento bioclimático}

\subsubsection{Ambiente Externo:}

Para a coleta das variáveis ambientais externas, utilizou-se a estação climatológica da Universidade Estadual Paulista (Unesp) Campus de Tupã, que se distanciava em 20km do município de Bastos-SP. Durante o período do estudo, foi analisado um ciclo produtivo de 21 dias no verão de 2013.

\subsubsection{Ambiente interno:}

Na coleta das variáveis bioclimáticas internas, empregou-se o uso de equipamentos do tipo data loggers (HOBO U12012 (Onset $\left.{ }^{\circledR}\right)$ ) com canal externo para sensor de temperatura/TMC50-HD no interior de cada aviário. Monitorou-se as seguintes variáveis: temperatura de bulbo seco (Tbs) e umidade relativa (UR), com 31 equipamentos de $30 \mathrm{em} 30$ minutos. A distribuição dos equipamentos ocorreu da seguinte forma: sete data loggers HOBO no aviário A1 e doze data loggers HOBO no galpão A2 e A3. Com estes registros foi possível calcular o índice de conforto térmico. 


\subsection{Descrição dos aviários}

\subsubsection{Funcionamento do aviário A1 (Piramidal):}

O sistema de produção, neste aviário, é do tipo piramidal, com três andares de gaiolas e duas baterias. A coleta de ovos foi realizada por esteira automática acoplada ao sistema, coletados uma vez ao dia. O arraçoamento foi realizado automaticamente, o fornecimento da água foi ad libitum e realizado por meio de bebedouros tipo nipple. As lâmpadas foram ligadas durante a madrugada entre uma hora e duas horas da manhã e depois das quatro horas e meia às sete horas da manhã, sendo aproveitada a iluminação natural a partir desse horário, e reacendendo às vinte horas até vinte e uma e meia horas, totalizando fotoperíodo de 17 horas de luz contínua, com 1 hora de luz intermitente. $O$ restante do tempo ficou destinado para o descanso das aves. Este galpão não possuía nenhum tipo de sistema de climatização e o esterco era retirado manualmente.

\subsubsection{Funcionamento do aviário A2 (Vertical climatizado):}

Foi, neste caso, utilizado um sistema de produção do tipo vertical, contendo três baterias com seis andares de gaiolas. A ração foi fornecida por comedouro automático, a coleta de ovos foi feita por esteiras automáticas conectadas ao sistema e a água ficou disponível em bebedouros ad libitum, três para cada duas gaiolas. A ração foi a mesma fornecida no galpão A1 devido as aves serem da mesma idade e linhagem. Nesse galpão foram instalados doze data loggers HOBO, distribuídos em três unidades por corredor. Neste galpão, o sistema de climatização foi realizado por exaustores e pad cooling (de celulose). Possuindo quatorze exaustores em uma extremidade do galpão e em outra o pad cooling presente também nas laterais direita e esquerda da extremidade. Quanto ao manejo das cortinas deste galpão, estas permaneceram fechadas durante o dia todo, devido ao sistema de climatização que exige total vedação do ambiente para o funcionamento do túnel adiabático e para que as condições internas não fossem afetadas pelas externas.

\subsubsection{Funcionamento do aviário A3 (Vertical sem climatização):}

O galpão A3 teve distribuição de gaiolas do tipo vertical e não possuía sistema de climatização sendo sua ventilação natural e possuía aspersão no telhado. A coleta de ovos e a retirada de esterco foram realizadas automaticamente. $\mathrm{O}$ arraçoamento foi realizado por comedouro automático, os bebedouros do tipo nipple e a água foi oferecida ad libitum. A dieta oferecida foi a mesma dos galpões A1 e A2. Havia duas baterias com seis andares de gaiolas, uma bateria formada por gaiolas de arame e a outra de polietileno. Neste galpão foram instalados doze Hobos.

\subsection{Cálculo de Viabilidade:}

Para se calcular a viabilidade (\%), o número de aves mortas foi anotado diariamente para a determinação da viabilidade total dos lotes (em função do número de aves alojadas), descrito pela equação 6.

$$
V=\frac{A t-A s}{A s} \times 100
$$

Equação 1: Viabilidade

Em que:

V: Viabilidade (\%);

At: Número de aves alojadas;

As: Número de aves mortas durante a semana.

\subsection{Cálculo de Entalpia:}

Para caracterizar a zona de conforto térmico adequada às aves, foi determinado o índice de conforto térmico Entalpia, 
por meio da fórmula citada por Furlan (2001). Os dados de $\mathrm{Tbs}^{\circ} \mathrm{C}$ e UR\% foram aplicados na fórmula, sendo possível assim calcular-se o índice de Entalpia (Equação 2):

$$
H=6,7+0,243 X T b s+\left(U R / 100 \quad \llbracket 10 \rrbracket^{\wedge}((7,5 \times T b s) /(237,3+T b s))\right)
$$

\section{Equação2: Entalpia}

Onde:

$\mathrm{H}$ : entalpia (kcal/kg ar seco);

Tbs: temperatura de bulbo seco $\left({ }^{\circ} \mathrm{C}\right) \mathrm{e}$

UR: umidade relativa do ar (\%).

Na Tabela 1 são apresentadas as faixas de classificação segundo os valores obtidos através da Equação 1(FURLAN, 2001).

Tabela 1 - Zonas de conforto de acordo com Entalpia.

\begin{tabular}{ccc}
\hline Faixas & Entalpia & Nível de estresse \\
\hline 1 & 56,1 a 64,6 & Conforto \\
2 & 64,7 a 70,5 & Alerta \\
3 & 70,6 a 77,5 & Crítica \\
4 & 77,6 a 93,1 & Letal \\
\hline
\end{tabular}

Fonte: Autores.

\subsection{Mineração de Dados:}

A segunda etapa consistiu na realização do processo de Mineração de Dados que foi dividido em fases distintas, sucedidos de forma cíclica, a fim de permitir novas considerações e reavaliações em relação ao domínio e à inclusão ou exclusão de atributos, de acordo com a metodologia proposta pelo consórcio CRISP-DM (Vale et al, 2008).

Os modelos induzidos com uma variação no número de instâncias (ou observações), por folha, foram avaliados usando o método de validação cruzada. Foi realizada a poda dos atributos que não contribuíram para os resultados de performance das árvores de decisão. A seleção do melhor modelo foi feita com base nas medidas: precisão; o número de folhas (regras) e o coeficiente Kappa.

O coeficiente Kappa é usado para descrever a medida de concordância entre as classes previstas e observadas. Esse coeficiente varia de 0 a 1, representando resultados de classificação muito ruins a excelentes, respectivamente (Lima et al., 2017). Como resultado da indução do modelo de árvore de decisão, foi possível obter a matriz de confusão, que segundo Han et al. (2011) é amplamente utilizada na análise estatística de concordância. A partir da matriz de confusão, segundo Lima et al. (2017), foi possível obter as medidas de avaliação de desempenho.

Após a organização dos dados em planilha de Excel® para cada aviário separadamente e respectivamente classificados de acordo com as faixas de Entalpia, construiu-se um arquivo em formato compatível com o software Weka® em extensão “.arff”. Estes arquivos organizados e classificados em formato “.arff”, foram executados no software Weka®. Escolheu-se o algoritmo J48 como procedimento de classificação, gerando após este processo a elaboração das saídas para a confeç̧ão das árvores de decisão. 


\section{Resultados e Discussão}

Após a inserção do arquivo em formato “.arff” no software Weka®, geraram-se árvores de decisão e valores de classificação e precisão das mesmas. A seguir, são apresentados os resultados para os três aviários na estação de verão.

\section{- Aviário piramidal (A1)}

Figura 1 - Resultados do algoritmo J48 com o sumário estatístico do processamento do conjunto de dados do aviário piramidal e resultados da precisão de classificação.

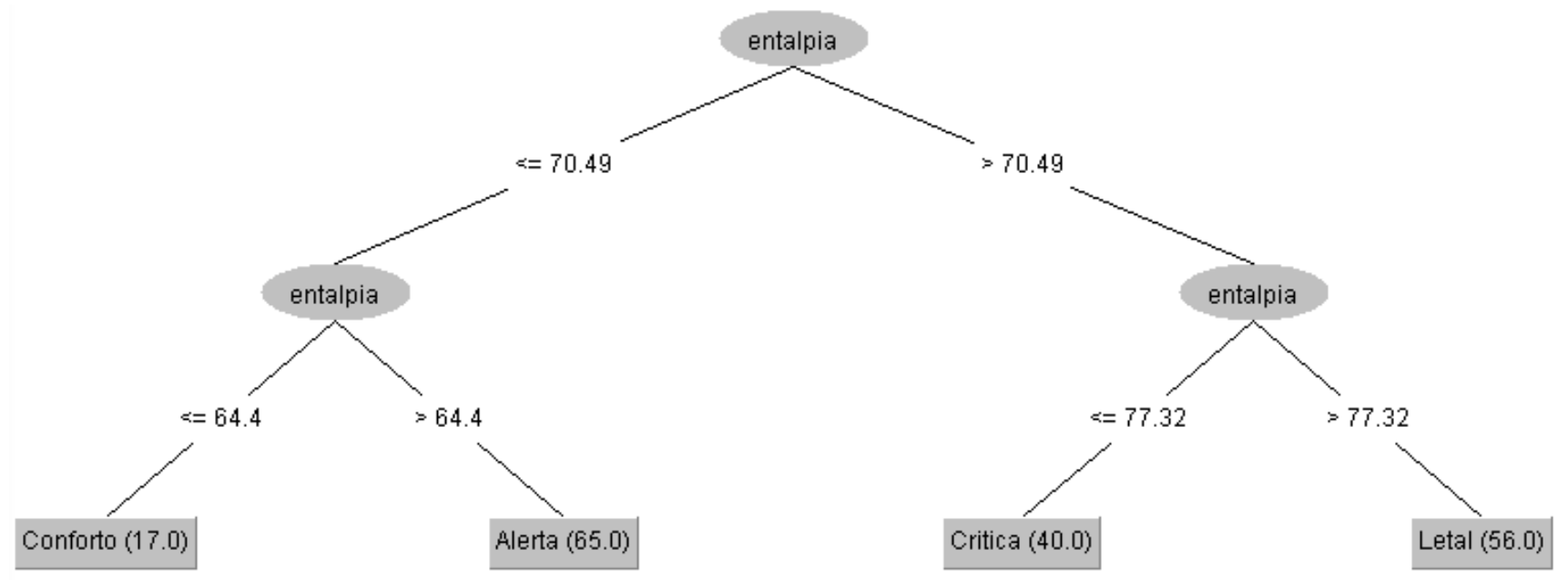

Fonte: Autores.

O algoritmo obtido como modelo de mineração de 98,3146\% e 0,9762 de índice Kappa, o que, segundo o método sugerido por Lima et al. (2017), torna-se possível qualificar a mineração como excelente.

\section{- Aviário vertical climatizado (A2)}

Figura 2 - Visualização da árvore de decisão gerada pela ferramenta de mineração de dados Weka® do aviário vertical climatizado.

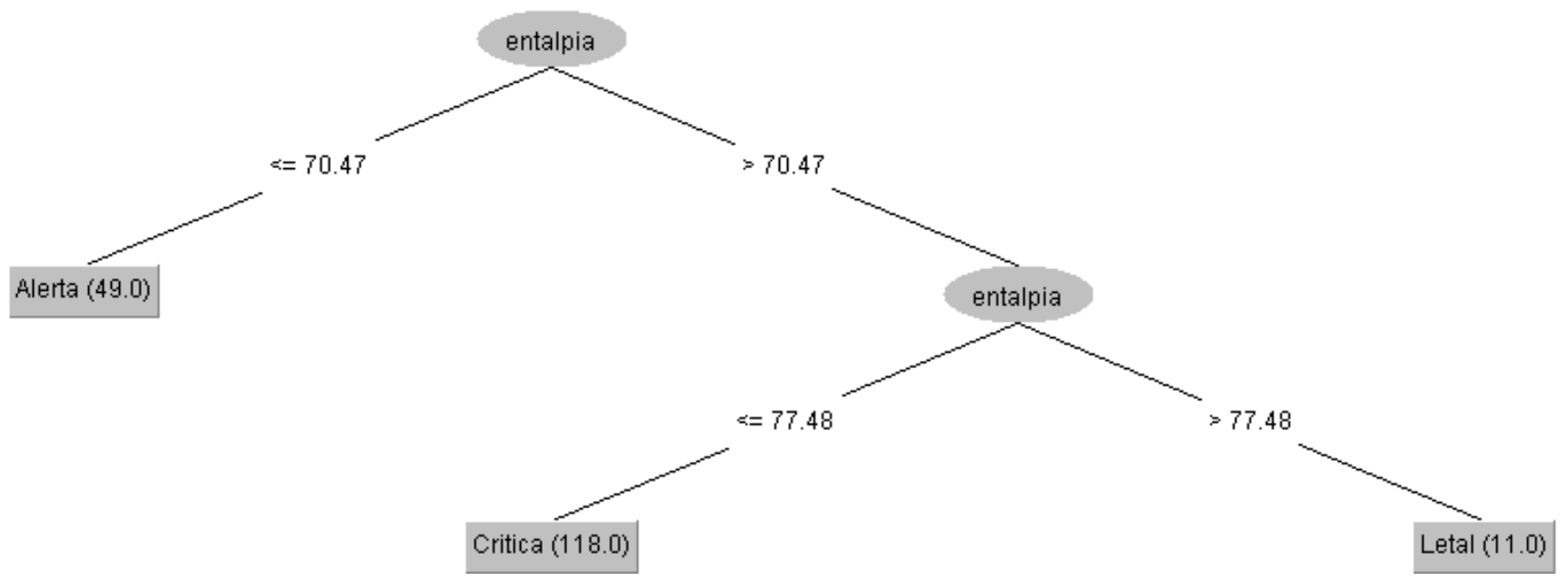

Fonte: Autores.

Com valores de 98,8764\% e 0,9767 para modelo de mineração e índice Kappa respectivamente, pode se considerar que 
a mineração de dados foi precisa, obtendo valores excelentes (Lima et al., 2017).

\section{• Aviário vertical não climatizado (A3)}

Figura 3 - Visualização da árvore de decisão gerada pela ferramenta de mineração de dados Weka ${ }^{\circledR}$ do aviário vertical não climatizado.

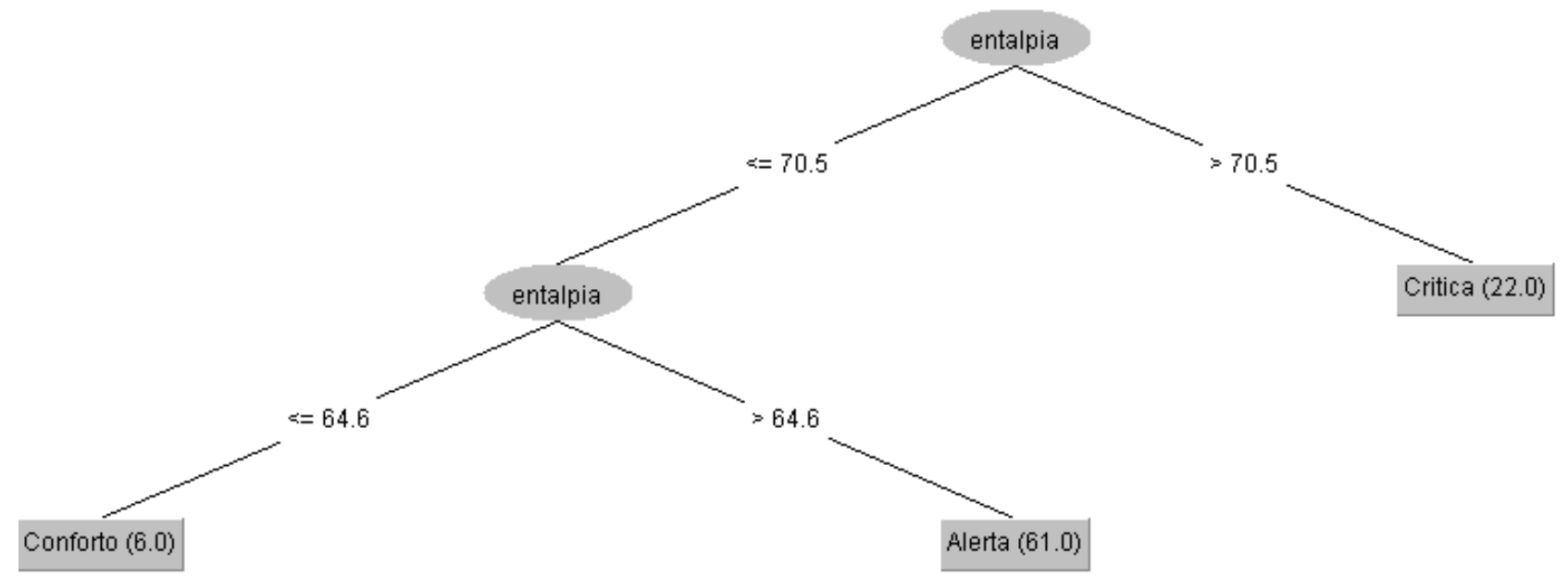

Fonte: Autores.

Para o aviário A3, a mineração de dados conforme a proposta de, foi classificada como excelente, pois o algoritmo de 97,7528\% e índice Kappa de 0,9514 são valores que nos permitem inferir com precisão os dados obtidos na árvore. É possível observar (Figura 1), que no aviário A1 (piramidal), foram encontrados todos os cenários possíveis, desde conforto térmico a letal. Por ser uma instalação que é dependente do ambiente externo, já era esperado que ocorresse uma situação de letalidade $(>77,32)$. Porém, mesmo sendo dependente de eventos climáticos externos para que haja as trocas de calor (Abreu et al., 2007) e consequentemente a melhoria dos valores de entalpia encontrados, a situação de conforto térmico foi encontrado $(\leq 64,4)$.

Quando analisado o aviário A2 (climatizado por pressão negativa) (Figura 2), existe uma disparidade em relação a proposta da tipologia construtiva e os valores de entalpia proporcionado para as poedeiras instaladas na mesma. O aviário A2 não foi capaz de proporcionar conforto térmico para as aves na estação do verão, os valores fornecidos pela árvore de decisão começam com o estado de alerta e segue até o cenário de letalidade ( $\leq 70,47$ e $\geq 77,48)$. Situação preocupante já que se trata de um aviário que possui tecnologias de climatização. Estes valores de entalpia mostram que existe a necessidade constante de manutenção do sistema, para que o mesmo alcance a eficiência almejada pelo projeto.

No aviário A3(vertical sem climatização), não houve cenário de letalidade durante a estação do verão. Esta instalação chegou apenas em situação entre conforto térmico $(\leq 64,6)$ e crítica $(>70,5)$, mesmo contando com o manejo de cortinas e aspersão de gotículas de água no telhado, um resultado positivo em relação ao aviário A2 em que se esperava um cenário apenas de conforto térmico.

Quando se comparam os três aviários, o que teve resultados mais interessantes e consequentemente conseguiu proporcionar melhor ambiente térmico para as aves, foi o A3. Além disso, este aviário apresentou uma viabilidade de 89,46\%, sendo melhor que o aviário A2 (88,46\%) e A1 (80,26\%). O A3 o melhor em relação a viabilidade e valores de Entalpia; todas as instalações ficaram a quém do recomendado pelo manual da linhagem $(95,90 \%)$ (Dekalb White $\left.{ }^{\circledR}, 2021\right)$. Esperava-se que o aviário A2 tivesse um melhor desempenho de todos, pois ele é de tipologia construtiva fechada e com sistema de climatização. É de conhecimento que estratégias construtivas como aspersão de água no telhado têm efeitos positivos em aviários. A aspersão 
de água na superfície de telhas de aço é capaz de reduzir aproximadamente $19,5^{\circ} \mathrm{C}$ de temperatura superficial, e até $1,9^{\circ} \mathrm{C}$ de temperatura próximo a altura da ave (Machado et al., 2016).

\section{Conclusão}

Com os resultados apresentados sobre este estudo, conclui-se que é possível gerar árvores de decisão com precisão para poedeiras a partir de dados de Entalpia. A mineração de dados mostrou-se eficaz na predição do conforto térmico. Após a validação das árvores de decisão, será possível o desenvolvimento de um software computacional para a classificação do conforto térmico e facilitar a tomada de decisão do produtor.

\section{Agradecimentos}

Os autores agradecem ao CNPq Processo n 313339/2019-8, à FAPESP Processo nº 2018/17447-1 e à CAPES processo $\mathrm{n}^{\mathrm{o}} 88887.480645 / 2020-00$ pelo apoio às pesquisas.

\section{Referências}

Abreu, P. G., Abreu, V. M. N., Coldebella, A., Jaenisch, F. R. F. \& Paiva, D. P. (2007). Condições térmicas ambientais e desempenho de aves criadas em aviários com e sem o uso de forro. Arquivo Brasileiro de Medicina Veterinária e Zootecnia, 59(4), 1014-20

Andrade, R. R., Tinoco, I. F. F., Souza, C. F., Oliveira, K. P., Barbari, M., Cruz, V. M. F., Baptista, F. J. F., Vilela, M. O., Conti, L., \& Rossi, G. (2018). Effect of thermal environment on body temperature of early-stage laying hens. Agronomy Research 16(2), 320327.

Anid, Associação Nacional Para Inclusão. (2015). Recuperado de http://www.anid.com.br/site//

Balnave, D., \& Brake, J. (2005). Nutrition and management of heat-stressed pullets and laying hens. World'S Poultry Science Journal, 61(3), $399-406$.

Celik, L. B., Tekeli, A., \& Ozturkcan, O. (2004). Effects of supplemental L-carnitine in drinking water on performance and egg quality of laying hens exposed to a high ambient temperature. Journal of Animal Physiology and Animal Nutrition, 88(5-6), 229-233.

Centro de Pesquisas Meteorológicas e Climáticas Aplicadas a Agricultura - CEPAGRI. (2017). Recuperado de http://www.cepagri.unicamp.br/outrasinformacoes/clima-dos-municipios-paulistas.html

Chung, U., Gbegbelegbe, S., Shiferaw, B., Robertson, R., Yun, J. I., Tesfaye, K., Hoogenboom, G., \& Sonder, K. (2014). Modeling the effect of a heat wave on maize production in the USA and its implications on food security in the developing world. Weather and Climate Extremes, 5(1), 67-77.

Damasceno, F. A., Junior, T. Y., Lima, R. R., Gomes, R. C. C., \& Moraes, S. R. P. (2010). Avaliação do bem-estar de frangos de corte em dois galpões comerciais climatizados. Ciência e Agrotecnologia, 34(4), 1031-1038.

Dekalb White®. (2021). https://www.dekalb-poultry.com/documents/137/DW_cs_c_leaflet_L7150_1_bpt.pdf

Etches, R. J., John, T. M., \& Verrinder Gibbins, A. M. (2008). Behavioural, physiological, neuroendocrine and molecular responses to heat stress. Pages 48-79 in Poultry Production in Hot Climates. (2a ed.), N. J. Daghir, ed. CAB International, Wallingford, UK.

Furlan, R.A. (2001). Avaliação da nebulização e abertura de cortinas na redução da temperatura do ar em ambiente protegido. Piracicaba: Escola Superior de Agricultura Luiz de Queiroz. 146p. Tese (Doutorado em Irrigação e Drenagem) - Escola Superior de Agricultura Luiz de Queiroz.

Guimarães, M. C. C., Furtado, D. A., Nascimento, J. W. B., Tota, L. C. A., Silva, C. M., \& Lopes, K. B. P. (2014). Efeito da estação do ano sobre o desempenho produtivo de codornas no semiárido paraibano. Revista Brasileira de Engenharia Agrícola e Ambiental, v.18, p.231-237.

Han, J., \& Kamber, M. (2006). Data mining: concepts and techniques. Morgan Kaufmann, (2a ed.), 2006,770 p.

Han, J., Kamber, M., \& Pei, J. (2011). Data Mining: concepts and techniques. (3a ed.), Morgan Kaufmann Publishers.

INMET- Instituto Nacional de Meteorologia (2020). Avisos meteorológicos Brasil, 2020. Recuperado de https://portal.inmet.gov.br/noticias/recorde-históricode-temperatura-nos-estados-do-mato-grosso-do-sul-e-são-paulo

Lara, L. J., \& Rostagno, M. H. (2013). Impact of heat stress on poultry production animals. Animals, 3(2), $356-369$.

Liao, S. H. (2003). Knowledge management technologies and applications - literature review from 1995 to 2002. Expert Systems with Applications, 25,' 155164.

Lima, E. S., Souza, Z. M., Montanari, R., Oliveira, S. R. M., Lovera, L. H., \& Farhate, C. V. V. (2017). Classification of the initial development of eucaliptus using data mining techniques. CERNE, Lavras, 23(2), 201-208. 
Research, Society and Development, v. 10, n. 3, e45310313354, 2021

(CC BY 4.0) | ISSN 2525-3409 | DOI: http://dx.doi.org/10.33448/rsd-v10i3.13354

Machado, N. S., Tinôco, I. F. F., Zolnier, S, Mogami, C. A., \& Rocha, K. S. O. (2016). Rain Water Spray Stored On The Broiler Sheds Cover With Steel Tiles In The Center West Region Of Brazil. Nucleus, 13(1), 153-168.

Medeiros, C. M., Baêta, F. C., Oliveira, R. F. M., Tinôco, I. F. F., Ablino, L. F. T., \& Cecon, P. R. (2005). Índice térmico ambiental de produtividade para frangos de corte. Revista Brasileira de Engenharia Agrícola e Ambiental, 9(4), 660-665.

Oliveira, D. R. M. S., \& Nääs, I. A. (2012). Issues of sustainability on the Brazilian broiler meat production chain. In: International Conference Advances In Production Management Systems. Rhodes. Anais. Competitive Manufacturing for Innovative Products and Services: proceedings, Greece: Internacional Federation for Information Processing.

Pereira, D. F., Vale, M. M., Zevolli, B. R., \& Salgado, D. D. (2010). Estimating mortality in laying hens as the environmental temperature increases. Brazilian Journal of Poultry Science, 12(4), 265-271.

Salgado, D. D., \& Nääs, I. A. (2010). Avaliação de risco à produção de frango de corte do Estado de São Paulo em função da temperatura ambiente. Engenharia Agrícola, 30(3), 367-376.

Seven, P. T. (2008). The Effects of Dietary Turkish Propolis and Vitamin C on Performance, Digestibility, Egg Production and Egg Quality in Laying Hens under Different Environmental Temperatures. Asian-Australasian Journal of Animal Sciences, 21(8), 1164-1170.

Sohail, M. U., Ijaz, A., Yousaf, M.s., Ashraf, K., Zaneb, H., Aleem, M., \& Rehman, H. (2010). Alleviation of cyclic heat stress in broilers by dietary supplementation of mannan-oligosaccharide and Lactobacillus-based probiotic: dynamics of cortisol, thyroid hormones, cholesterol, c-reactive protein, and humoral immunity. Poultry Science, 89(9), 1934-1938.

Teeter, R. G., Smith, M. O., Owens, F. N., ARP, S. C., Sangiah, S., \& Breazile, J.e. (1985). Chronic Heat Stress and Respiratory Alkalosis: occurrence and treatment in broiler chicks. Poultry Science, 64(6), 1060-1064.

Vale, M. M., Moura, D. J., Nääs, I. A., Oliveira, R. M., \& Rodrigues, L. H. A. (2008). Data mining to estimate broiler mortality when exposed to heat wave. Scientia Agricola (Piracicaba, Braz.), .65(3), 223-229.

Yahav, S., Straschnow, A., Luger, D., Shinder, D., Tanny, J., \& Cohen, S. (2004). Ventilation, sensible heat loss, broiler energy, and water balance under harsh environmental conditions. Poultry Science, 83(2), 253-258. 